\title{
Skin Cancer Detection using Digital Image Processing and Implementation using ANN and ABCD Features
}

\author{
Khaing Thazin Oo ${ }^{1}$, Dr. Moe Mon Myint ${ }^{2}$, Dr. Khin Thuzar Win ${ }^{3}$ \\ ${ }^{1}$ Assistant Lecturer, ${ }^{2,3}$ Professor \\ ${ }^{1,2}$ Department of Electronics Engineering, ${ }^{3}$ Department of Mechronics Engineering, \\ Pyay Technological University, Myanmar
}

\begin{abstract}
Melanoma is a serious type of skin cancer. It starts in skin cells called melanocytes. There are 3 main types of skin cancer, Melanoma, Basal and Squamous cell carcinoma. Melanoma is more likely to spread to other parts of the body. Early detection of malignant melanoma in dermoscopy images is very important and critical, since its detection in the early stage can be helpful to cure it. Computer Aided Diagnosis systems can be very helpful to facilitate the early detection of cancers for dermatologists. Image processing is a commonly used method for skin cancer detection from the appearance of affected area on the skin. In this work, a computerised method has been developed to make use of Neural Networks in the field of medical image processing. The ultimate aim of this paper is to implement cost-effective emergency support systems; to process the medical images. It is more advantageous to patients. The dermoscopy image of suspect area of skin cancer is taken and it goes under various pre-processing technique for noise removal and image enhancement. Then the image is undergone to segmentation using Thresholding method. Some features of image have to be extracted using ABCD rules. In this work, Asymmetry index and Geometric features are extracted from the segmented image. These features are given as the input to classifier. Artificial Neural Network (ANN) with feed forward architecture is used for classification purpose. It classifies the given image into cancerous or non-cancerous. The proposed algorithm has been tested on the ISIC (International Skin Imaging Collaboration) 2017 training and test datasets. The ground truth data of each image is available as well, so performance of this work can evaluate quantitatively.
\end{abstract}

Keyword: Skin cancer, Segmentation, Feature Extraction, Classification, Melanoma

\section{INTRODUCTION}

Skin cancers can be classified into melanoma and non-melanoma. Melanoma is a malignancy of the cells which gives the skin its colour (melanocytes) and it can invade nearby tissues. Moreover, it spreads through the whole human body and it might cause to patient death and non-melanoma which is rarely spread to other parts of the human body. Malignant melanoma is the most aggressive type of human skin cancers and its incidence has been rapidly increasing [1] [2] [3] [4]. Nevertheless, it is also the most treatable type of skin cancer if detected or diagnosed at an early stage [5]. The diagnosis of melanoma in early stage is a challenging and fundamental task for dermatologists since some other skin lesions may have similar physical characteristics. Dermoscopy is considered as the widely common technique used to perform an in-vivo observation of pigmented skin lesions [6]. In early detection of malignant melanoma, dermoscopic images have great potential, but their interpretation is time consuming and subjective, even for trained dermatologists. Therefore, the need to build a system which can assist dermatologists to get right decision for their diagnosis has become very important.

Image processing is one of the widely used methods for skin cancer detection. Dermoscopy could be a non-invasive examination technique supported the cause of incident light beam and oil immersion technique to form potential the visual investigation of surface structures of the skin. The detection of melanoma using dermoscopy is higher than individual observation based detection [3], but its diagnostic 
accuracy depends on the factor of training the dermatologist.

The diagnosis of melanoma from melanocytic nevi is not clear and easy to identify, especially in the early stage. Thus, automatic diagnosis tool is more effective and essential part of physicians. Even when the dermoscopy for diagnosis is done with the expert dermatologists, the accuracy of melanoma diagnosis is not more than $75-84 \%$ [4]. The computer aided diagnostics is more useful to increase the diagnosis accuracy as well as the speed [5].

The computer is not more inventive than human but probably it may be able to extract some information, like colour variation, asymmetry, texture features, more accurately that may not be readily observed by naked human eyes [5]. There have been many proposed systems and algorithms such as the sevenpoint checklist, ABCD rule, and the Menzies method $[2,3]$ to improve the diagnostics of the melanoma skin cancer.

The key steps in a computer-aided diagnosis of melanoma skin cancer are image acquisition of a skin lesion, segmentation of the skin lesion from/skin region, extraction of geometric features of the lesion blob and feature classification. Segmentation or border detection is the course of action of separating the skin lesion of melanoma from the circumferential skin to form the area of interest. Feature extraction is done to extract the geometric features which are accountable for increasing the accuracy; corresponding to those visually detected by dermatologists, that meticulously characterizes a melanoma lesion.

The feature extraction methodology of many computerised melanoma detection systems has been largely depending on the conventional clinical diagnostic algorithm of ABCD-rule of dermoscopy due to its effectiveness and simplicity of implementation [7]. The effectiveness of methodology stems from the fact that it incorporates the classic features of a melanoma lesion such as asymmetry, border irregularity, colour and diameter (or differential structures), where surveyable measures can be computed.

Dermoscopy is a diagnostic technique that is used worldwide in the recognition and interpretation of copious skin lesions [4]. Other than dermoscopy, a computerised melanoma detection using Artificial Neural Network classification has been adapted which is efficient than the conventional one and Melanoma detection using Artificial Neural Network is a more effective method compared to other.

\section{Methodology}

The following steps are implemented for classification of skin cancer.

$>$ Image Acquisition

$>$ Pre-processing

$>$ Segmentation

$>$ Feature Extraction

$>$ Classification

The first step is the capture image the skin lesion image is acquired from the ISIC 2018 database through MATLAB. The second step is image preprocessing, the pre-processing technique can be applied to eliminate the irrelevant data contains in the image. The skin lesion regions such as label, marks, oils, hairs are removed from the original image using median filter techniques. The third step is image segmentation: the goal of image segmentation is to make simpler change the representation of an image into something that is more meaningful and easier to analyze. The next step is image enhancement; to improve the quality of the image so that the consequential image is better than the original image. In this step, the required region is segmented out and detected the edge of the skin lesion. The fourth step is feature extraction and the final step is classification of the skin lesion image.

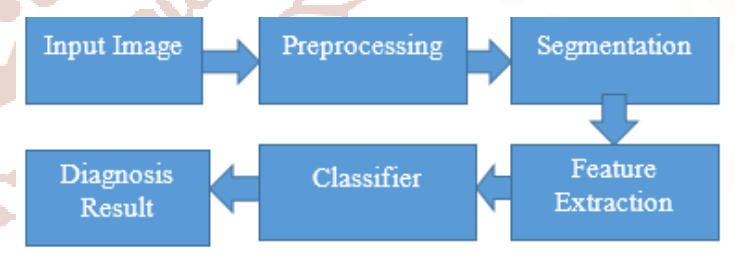

Fig. 1System Block Diagram image

\section{A. Pre-processing}

In this work, few previous processing techniques are needed. Prior to segmentation, all images are preprocessed in order to minimize undesirable features that could affect the performance of the algorithm such as reflections, presence of the hair and colour differences between images. The image is also normalized to a unique size and shape. This normalization allows for the comparison of the region features such as positions and sizes between different images. 


\section{B. Skin Lesion Segmentation}

Image segmentation is an essential process for most image analysis subsequent tasks. Segmentation divides an image into its constituent regions or objects. The goal of segmentation is to make simpler or change the representation of an image into something that is more meaningful and easier to analyse.

Image segmentation is the course of action of segregating an image into multiple parts, which is used to identify objects or other relevant information in digital images. Background subtraction, also known as blob detection, is an emerging technique in the fields of image processing wherein an image's foreground is extracted for further processing. Typically, an image's regions of interest are objects in its foreground. Segmentation methods can be classified as thresholding, region based, Edge based and clustering. In this work, thresholding is used because of the computationally inexpensive and fast and simple to implement.

\section{Post-processing}

Once binary image of skin lesion has been obtained, the image then needs to be post processed in order to resolve any problems there may be as follows:

$>$ To prevent edges which are dark in many of the images, from remaining as part of the lesion mask;

$>$ To reduce any effects of disturbing artifacts as far as possible;

To set soft edges, to ensure there are not too many recesses or projections and that there is certain convexity in the resulting mask

These problems can be reduces by using morphological operation such as opening, closing and filling process.

\section{Feature Extraction}

The foremost features of the Melanoma Skin Lesion are its Asymmetric Index, Border features, Colour and Diameter. Hence, this system is proposed to extract the (9) Geometric Features, (1) Asymmetry Index and (1) diameter of the segmented skin lesion. These features are adopted from the segmented image containing only skin lesion, the image blob of the skin lesion is analyzed to extract the 11 features.

Asymmetry Features: Major and Minor Asymmetry Indices:

Geometric Features:
1. Area (A): Number of pixels of the lesion.

2. Perimeter (P): Number of pixels along the detected boundary

3. Greatest Diameter (GD): The length of the line which connects the two farthest

4. Shortest Diameter (SD): The length of the line connecting the two closest boundary points and passes across the lesion centroid.

5. Circularity Index (CRC): It explains the shape uniformity.

6. Irregularity Index A (IrA):

7. Irregularity Index $B(\operatorname{IrB})$ :

8. Irregularity Index $C(\operatorname{IrC})$ :

9. Irregularity Index D (IrD):

Diameter: Diameter in pixels

\section{E. Classification}

The main issue of the classification task is to avoiding over fitting caused by the small number of images of skin lesion in most dermatology datasets. In order to solve this problem, the objective of the proposed model is to firstly extract features from images and secondly load those extracted representations on an ANN network to classify.

\section{Performance Evaluation Parameter}

The most common performance measures consider the model's ability to discern one class versus all others. The class of interest is known as the positive class, while all others are known as negative. The relationship between positive class and negative class predictions can be depicted as a $2 \times 2$ confusion matrix that tabulates whether predictions fall into one of four categories:

$>$ True positives (TP): These refer to the positive tuples that were correctly labelled by the classifier. It is assumed that TP is the number of true positives.

True negatives $(\mathrm{TN})$ : These are the negative tuples that were correctly labelled by the classifier. It is assumed that TN is the number of true negatives. False positive (FP): These are the negative tuples that were incorrectly labelled as positive. It is assumed that FP is the number of false positives.

False negative (FN): These are the positive tuples that were mislabelled as negative. It is assumed that $\mathrm{FN}$ is the number of false negatives.

Accuracy can be calculated by using this equation:

$$
\text { Accuracy }=\frac{\text { Number of correct predictions }}{\text { Total number of predictions }}
$$


International Journal of Trend in Scientific Research and Development (IJTSRD) ISSN: 2456-6470

$$
=\frac{\mathrm{TP}+\mathrm{TN}}{\mathrm{TP}+\mathrm{TN}+\mathrm{FP}+\mathrm{FN}}
$$

As indicated in Equation (1), accuracy only measures the number of correct predictions of the classifier and ignores the number of incorrect predictions.

Sensitivity, also known as recall, is computed as the fraction of true positives that are correctly identified.

$$
\text { Sensitivity }=\frac{\mathrm{TP}}{\mathrm{TP}+\mathrm{FN}}
$$

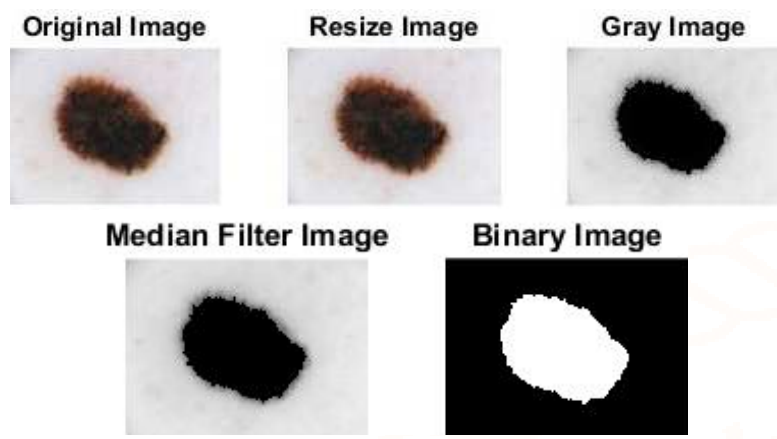

Fig.2. Testing result of pre-processing and segmentation

Precision, which is computed as the fraction of retrieved instances that are relevant.

$$
\text { Precision }=\frac{\mathrm{TP}}{\mathrm{TP}+\mathrm{FP}}
$$

Specificity, computed as the fraction of true negatives that are correctly identified.

$$
\text { Specificity }=\frac{\mathrm{TN}}{\mathrm{TN}+\mathrm{FP}}
$$

\section{Test And Results}

\section{A. Results of Segmentation Process}

There are three main phases: namely pre-processing, segmentation and post-processing in segmentation part. The input of the system is dermoscopic image of skin lesion as shown in Fig. 2. After resizing the input image, this image will convert to the gray scale image in order to get grater separability between the lesion and background healthy skin. The resultant gray scale image has been displayed. Most of the dermoscopic images have some artifacts such as oil, bubble hair, noise etc. these artifacts are removed by applying median filter on gray scale image. After this, the Otsu's thresholding method has been applied on the gray scale intensity image. This gives the desired segmented image.

After these steps morphological operations are used to enhance the segmented image. In this research work, morphological opening, dilation, erosion and closing operations are used. A morphological closing of the mask is performed using a disk of radius 500 pixels followed by a dilation using a disk of radius 40 pixels. These operations smooth the border of the mask. The experimental results for this post-processing phase are shown in Fig. 3.

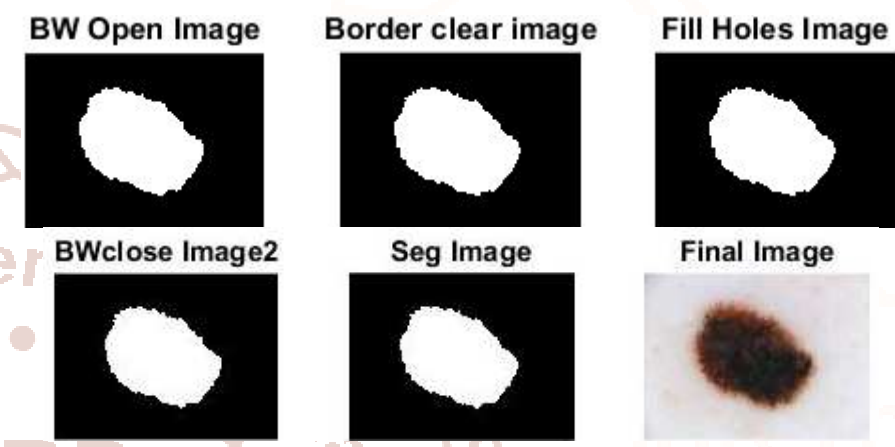

Fig.3. Testing result of segmented image after dilation and erosion

\section{B. Results of Classification}

The first stage of the system is to select skin lesion images. This can click original image menu item from main window GUI of the proposed system.

After loading the input image, it is needed to segment the lesion image by using Otsu's Thresholding Technique. The segmented image obtained from Otsu's thresholding has the advantages of smaller storage space, fast processing speed and ease in manipulation, compared with gray level image which usually contains 256 levels.

In feature extraction, the standard features such as Asymmetry Index, Area, Perimeter, Major Axis Length, Minor Axis length, Circularity Index, Irregularity Index and diameter are extracted from the segmented test image as shown in Fig.6. These standard features are very useful to classify the melanoma skin cancer more accurately.

After extracting the features, these features are given as input to the classifier. The classifier produces whether the image is Melanoma or not. For the Melanoma condition, the classifier output is 1 and for normal skin (not melanoma) the output is 0. By pressing the classify button, the classification process is performed and the result is displayed as shown in Fig. 7 and 8. 


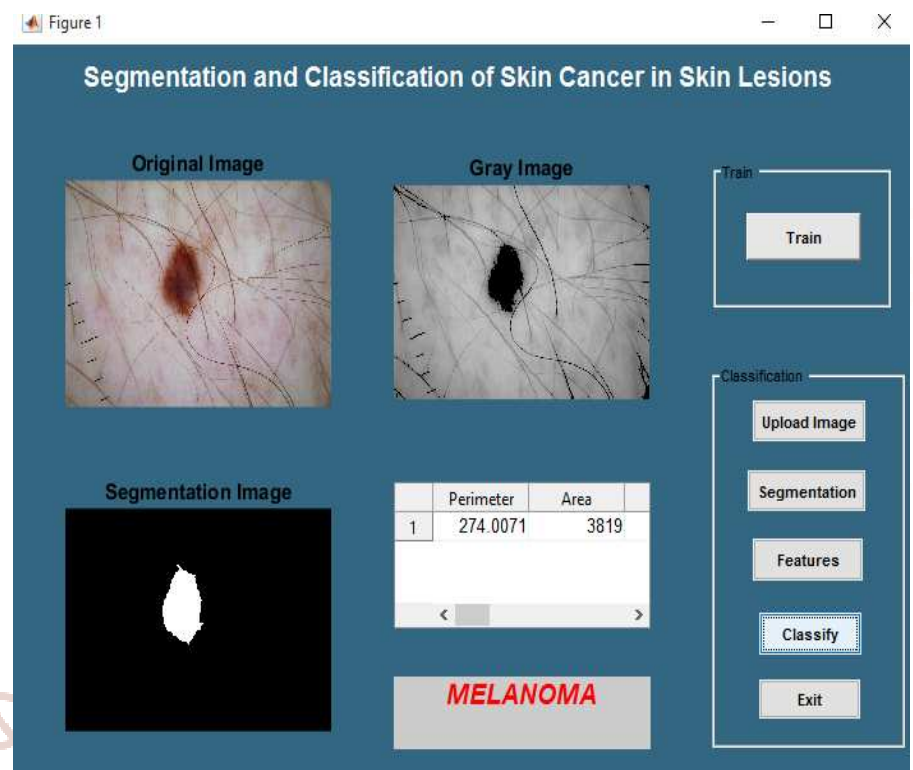

Fig.7. Classification Results of Melanoma

Fig.4. Loading Input Image

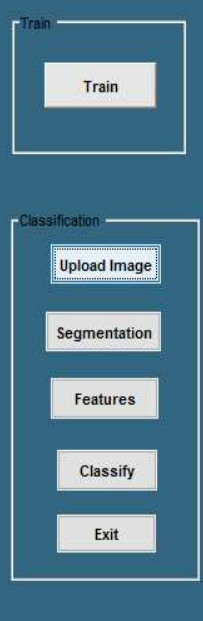

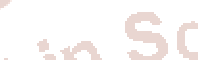

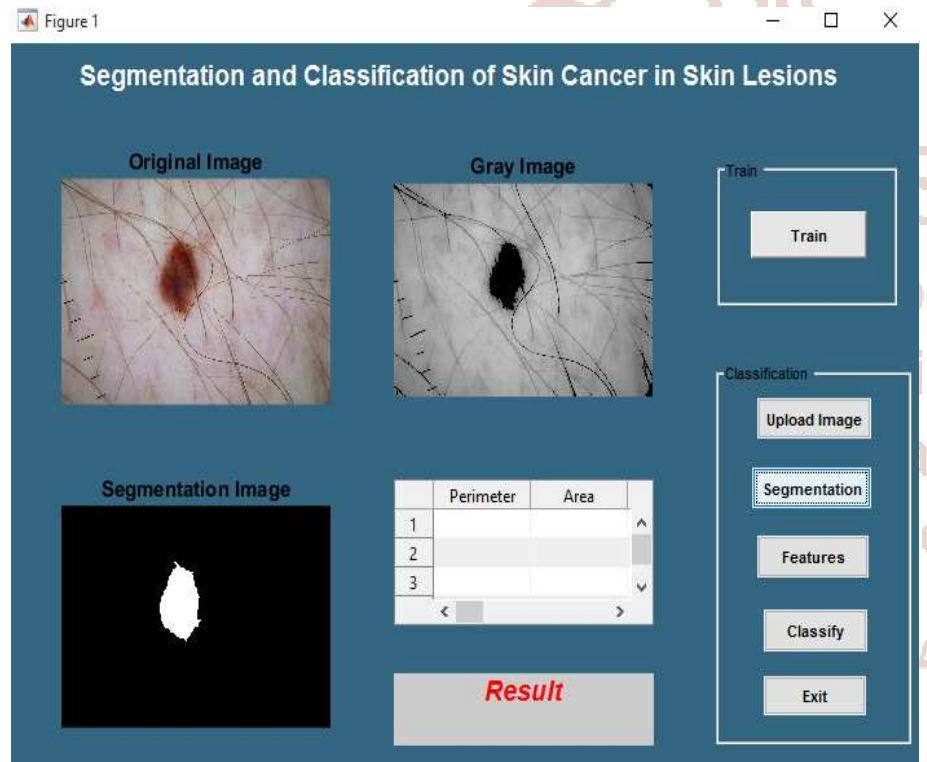

Fig.5. After Segmentation

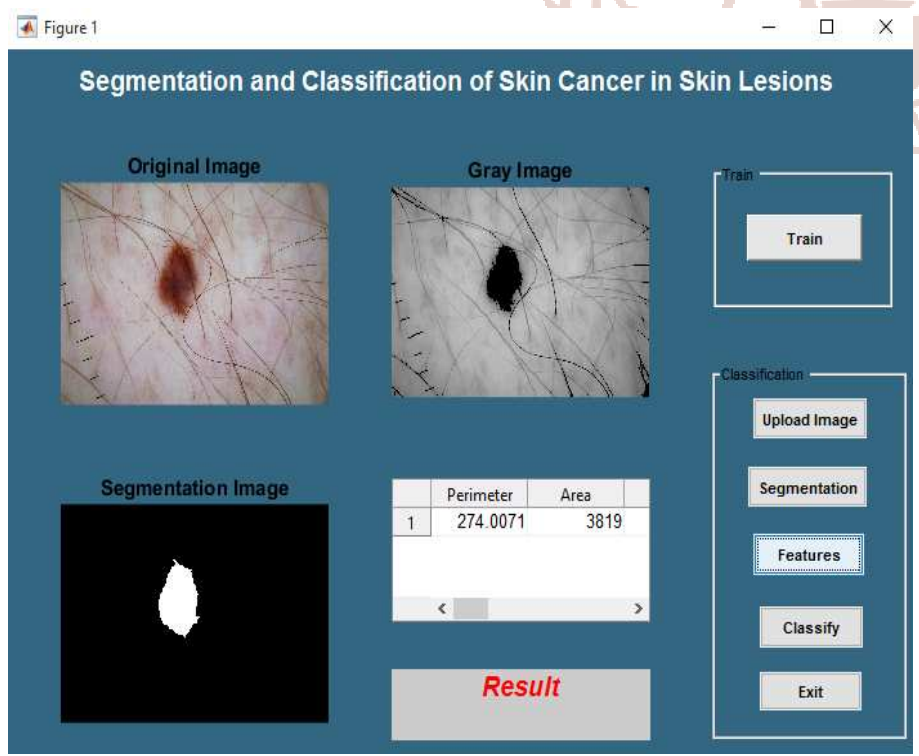

Fig.6. Extracted Features from Testing Image

\section{A. Figure 1}

Segmentation and Classification of Skin Cancer in Skin Lesions
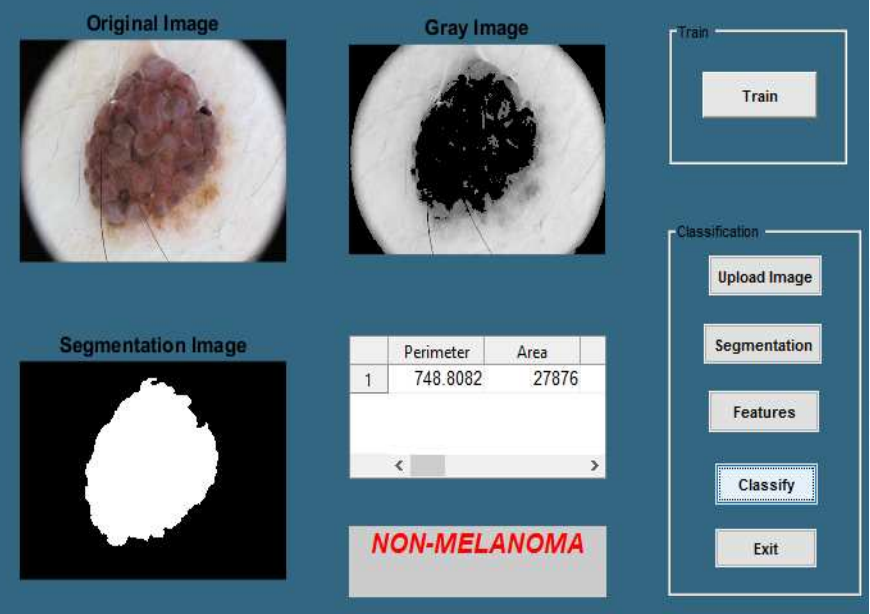

Fig.8. Classification Results of Non-Melanoma

\section{Performance Evaluation}

To evaluate performance in this system, 40 unknown images from a test data set are tested. The accuracy of skin lesion classification system is calculated. Table 1 describes the performance of the proposed system.

\section{TABLE I Performance of The Proposed System}

\begin{tabular}{|c|c|c|c|c|c|c|c|c|c|}
\hline $\begin{array}{l}N \\
0\end{array}$ & $\begin{array}{l}\text { Im } \\
\text { age } \\
\text { Set }\end{array}$ & TP & $\begin{array}{l}\mathrm{F} \\
\mathrm{P}\end{array}$ & $\begin{array}{l}\mathbf{T} \\
\mathbf{N}\end{array}$ & $\begin{array}{l}\mathrm{F} \\
\mathrm{N}\end{array}$ & $\begin{array}{c}\text { Acc } \\
\text { urac } \\
\mathbf{y}\end{array}$ & $\begin{array}{l}\text { Sensi } \\
\text { tivity }\end{array}$ & $\begin{array}{l}\text { Pre } \\
\text { cisi } \\
\text { on }\end{array}$ & $\begin{array}{l}\text { Spe } \\
\text { cific } \\
\text { ity }\end{array}$ \\
\hline 1 & $\begin{array}{c}\text { Tes } \\
\mathrm{t} \\
\text { ing } \\
\text { Set }\end{array}$ & 18 & 2 & 19 & 1 & $\begin{array}{c}92.5 \\
\%\end{array}$ & $\begin{array}{c}94.7 \\
\%\end{array}$ & $\begin{array}{l}90 \\
\%\end{array}$ & $\begin{array}{c}90.4 \\
7 \%\end{array}$ \\
\hline
\end{tabular}




\section{Conclusions}

In this work, the system for melanoma skin cancer detection system is developed by using MATLAB. Image segmentation is the first step in early detection of melanoma skin cancer. To analyze skin lesions, it is necessary to accurately locate and isolate the lesions. In this thesis work, the Otsu's method is the oldest and simplest one. It has shown the best segmentation results among the three methods. Feature extraction is considered as the most critical state-of-the- art skin cancer screening system. In this thesis, the feature extraction is based on ABCD-rule of dermatoscopy. Algorithms for extracting features have been disused. All the features have been calculated based on Otsu's segmentation method. Using the Neural Network classifier, melanoma skin cancer diagnosis with a training accuracy of $100 \%$ and testing accuracy of $93 \%$ is achieved. Computational time is around 14 seconds for each lesion classification. For illustration, a graphical user interface has developed in order to facilitate the diagnostic task for the dermatologists.

\section{Acknowledgment}

Firstly, the author would like to acknowledge particular thanks to Union Minister of the Ministry of Science and Education, for permitting to attend the Master program at Pyay Technology University. Much gratitude is owed to Dr. Nyaunt Soe, Rector, Pyay Technological University, for his kind permission to carry out this paper. The author is deeply thankful to her supervisor, Dr. Moe Mon Myint, Professor, Department of Electronic Engineering, Pyay Technological University, for her helpful and for providing guidelines. Moreover, the author wishes to express special thanks to Dr. Khin Thu Zar Win, Professor and Head, Department of Mechatronic Engineering, Pyay Technological University, Pyay for her kindness and suggestions. Finally, I would like to thank my parents for supporting to me.

\section{References}

1. Xu L, Jackowski M, Goshtasby A, Roseman, "Segmentation of cancer images", Image and Vision Computing, Volume 17, Issue1, pg. 65-74.

2. J Abdul Jaleel, Sibi Salim, Aswin. R. B, "Computer Aided Detection 01 Skin Cancer", International Conference on Circuits, Power and Computing Technologies, 2013

3. Arroyo J L G and Zapirain B G, "Computer Aided Detection 01 Skin Cancer", 2014, Computers in Computers in biology and .medicine 4414157 biology and medicine 4414157

4. Dr. S. Gopinathan, S. Nancy Arokia Rani, "Feature Extraction through Image Processing Techniques", International Journal of Emerging Trends \& Technology in Computer Science (IJETTCS), Volume 5, Issue 4, July-August 2016, Web Site:www.ijettcs.org

5. Uzma Bano Ansari, "Skin Cancer Detection Using Image Processing", International Research Journal of Engineering and Technology (IRJET), Volume: 04 | Apr-2017

6. Shivangi jain, Vandana jagtap, Nitin Pise, "Computer Aided Melanoma Skin Cancer Detection Using Image Processing", Internal Conference on Intelligent Computing Communication \& Convergence, Vol 48, pp 725740, 2015, ISSN 1877-0509, http://www.sciencedirect.com/science/article/pii/S 1877050915007188

7. M. Elbaum, "Computer-aided melanoma diagnosis", Dermatologic clinics, vol. 20, pp. 735747, 2002 